\title{
BOUND ON THE EXTREME ZEROS OF ORTHOGONAL POLYNOMIALS
}

\author{
MOURAND E. H. ISMAIL AND XIN LI
}

(Communicated by Kenneth R. Meyer)

\begin{abstract}
Using chain sequences we formulate a procedure to find upper (lower) bounds for the largest (smallest) zero of orthogonal polynomials in terms of their recurrence coefficients. We also apply our method to derive bounds for extreme zeros of the Laguerre, associated Laguerre, Meixner, and MeixnerPollaczek polynomials. In addition, we consider bounds for the extreme zeros of Jacobi polynomials of degree $n$ and parameters $a_{n}$ and $b_{n}$.
\end{abstract}

\section{INTRODUCTION}

Let $\left\{p_{n}(x)\right\}_{0}^{N}$ be a family of real polynomials recursively generated by

$$
\begin{gathered}
p_{0}(x)=1, \quad p_{1}(x)=\left(x-\beta_{0}\right) / \alpha_{0}, \\
x p_{n}(x)=\alpha_{n} p_{n+1}(x)+\beta_{n} p_{n}(x)+\gamma_{n} p_{n-1}(x), \quad 0<n<N
\end{gathered}
$$

and assume that the $\beta_{n}$ 's are real and that the positivity condition is

$$
\alpha_{n-1} \gamma_{n}>0, \quad 0<n<N \text {. }
$$

It is well known that $p_{n}(x)$ is a constant multiple of the characteristic polyno. mial of the tridiagonal matrix

$$
A_{n}=\left[\begin{array}{ccccc}
\beta_{0} & \alpha_{0} & 0 & \ldots & 0 \\
\gamma_{1} & \beta_{1} & \alpha_{1} & \ldots & 0 \\
\cdots & \cdots & \cdots & \ldots & \ldots \\
0 & 0 & \cdots & \gamma_{n-1} & \beta_{n-1}
\end{array}\right]
$$

Indeed

$$
\alpha_{0} \alpha_{1} \cdots \alpha_{n-1} p_{n}(x)=\operatorname{det}\left(I_{n} x-A_{n}\right), \quad n=1,2, \ldots, N .
$$

Recall that a sequence $\left\{a_{n}\right\}_{1}^{N}$ is a (finite) chain sequence if and only if there exists a parameter sequence $\left\{g_{n}\right\}_{0}^{N}$ such that the $a_{n}$ 's admit a factoring (1.3)

$$
\alpha_{n}=g_{n}\left(1-g_{n-1}\right), \quad 0<n \leq N, 0 \leq g_{0}<1,0<g_{n}<1,0<n \leq N .
$$

Received by the editors September 21, 1990 and, in revised form, November 12, 1990.

1980 Mathematics Subject Classification (1985 Revision). Primary 33A65; Secondary 12D10.

Key words and phrases. Bounds, chain sequences, Chihara-Wall-Wetzel theorem, Laguerre polynomials, largest zero, Meixner polynomials, Meixner-Pollaczek polynomials, recurrence relations, smallest zero.

The research was partially supported by NSF grants DMS 8814026 and DMS 8912423 . 
The applications treated here require $N$ to be finite in contrast with the approach in $[7,8]$ that uses only the case $N=\infty$.

It was pointed out in [14] that by using row reduction to row echelon form one can show that $A_{N}$ is positive definite if and only if $0<\beta_{n}, 0 \leq n<N$, and $\left\{\gamma_{n} \alpha_{n-1} / \beta_{n-1} \beta_{n}\right\}_{1}^{N-1}$ is a chain sequence. The latter result is a theorem of Wall and Wetzel [18], whose original proof used quadratic forms. Another proof of the Wall-Wetzel Theorem is in [7, 8]. The Wall-Wetzel Theorem implies the following theorem [7,8].

Theorem 1. Let $\left\{p_{n}(x)\right\}_{0}^{N}$ be as in (1.1) and assume positivity condition (1.2). The zeros of $p_{N}(x)$ (i.e., the eigenvalues of $\left.A_{N}\right)$ belong to $(A, B)$ if and only if

(i) $A<\beta_{n}<B, 0 \leq n<N$,

(ii) $\left\{\gamma_{n} \alpha_{n-1} /\left[\left(x-\beta_{n-1}\right)\left(x-\beta_{n}\right)\right]\right\}_{1}^{N-1}$ is a chain sequence at $x=A$ and $x=B$.

The purpose of this work is to prove Theorem 2 and apply it to obtain sharp upper (lower) bounds for the largest (smallest) zero of $p_{N}(x)$.

Theorem 2. Let $\left\{a_{n}\right\}_{1}^{N-1}$ be a chain sequence, and set

$$
B:=\max \left\{x_{n}: 0<n<N\right\} \quad \text { and } A:=\min \left\{y_{n}: 0<n<N\right\} \text {, }
$$

where $x_{n}$ and $y_{n}, x_{n} \geq y_{n}$, are the roots of the equation

$$
\left(x-\beta_{n}\right)\left(x-\beta_{n-1}\right) a_{n}=\gamma_{n} \alpha_{n-1} ;
$$

that is,

$$
y_{n}, x_{n}=\frac{1}{2}\left(\beta_{n}+\beta_{n-1}\right) \pm \frac{1}{2} \sqrt{\left(\beta_{n}-\beta_{n-1}\right)^{2}+4 \gamma_{n} \alpha_{n-1} / a_{n}} .
$$

Then the zeros of $p_{N}(x)$ lie in $(A, B)$.

Note that the roots of (1.5) are always real when the $\beta_{n}$ 's are real and positivity condition (1.2) is satisfied.

If $\left\{a_{n}\right\}_{1}^{N}$ is a chain sequence and $a_{n} \leq b_{n}$ for $0<n \leq N$, then $\left\{b_{n}\right\}_{1}^{N}$ is a chain sequence $[8,14]$. The choice of a large chain sequence $\left\{a_{n}\right\}$ improves the bounds in (1.4). This suggest the desirability of characterizing the largest constant finite chain sequence.

Theorem 3. A constant sequence $\{a\}_{1}^{N}$ is a finite chain sequence if and only if

$$
0<a<\left[4 \cos ^{2}(\pi /(N+2)]^{-1} .\right.
$$

The limiting case $N \rightarrow \infty$ of Theorem 3 is in Chihara [7], where (1.6) must be replaced by $0<a \leq 1 / 4$.

Note that Theorem 2 is an immediate consequence of Theorem 1 . In $\S 2$ we prove Theorem 3. Section 3 contains applications of Theorems 2 and 3 to certain orthogonal polynomials. We give upper bounds for the largest zeros of Meixner, Laguerre, and associated Laguerre polynomials. We also give lower bounds for the smallest zeros of the same polynomials. In addition we compare our estimates with the known estimates of the extreme zeros of Laguerre polynomials. In $\S 4$ we apply Theorem 2 to the case of Meixner-Pollaczek polynomials and find bounds for the largest zeros of Pollaczek polynomials. Our estimates for the Meixner, Meixner-Pollaczek, and associated Laguerre polnomials are 
new. We determine precisely the large $N$ behaviour of the largest zero of a Meixner, Meixner-Pollaczek, and an associated Laguerre polynomial of degree $N$ and show that our bounds are sharp. It may be of interest to point out that the classical bounds on extreme zeros of the Jacobi, Hermite, and Laguerre polynomials use the very refined theory of second order differential equations of Sturm-Liouville type. Such theory does not apply to associated Laguerre nor the Meixner polynomials. In fact very few orthogonal polynomials satisfy second order differential equations of Sturm-Liouville type $[1,8]$. On the other hand every family (finite or infinite) of orthogonal polynomials satisfies a three term recurrence relation of the type in (1.1).

In $\S 5$ we derive upper and lower bounds for the largest and smallest zeros, respectively, of the Jacobi polynomials $P_{n}^{\left(a_{n}, b_{n}\right)}(x)$ when $a_{n}>-1$ and $b_{n}>-1$. This sharpens a result of Moak, Saff, and Varga [15] derived in conjunction with the sharpness of Lorentz's theorem on approximation by incomplete polynomials. When $a_{n}=a n+b$ and $b_{n}=c n+d$, Chen and Ismail [6] obtained strong asymptotics for the corresponding Jacobi polynomials and for Laguerre polynomials $L_{n}^{(a n+b)}(x+c n)$. Gawronski [10] independently obtained strong asymptotics for the special case $c=0$ of the aforementioned Laguerre polynomials. Recently Gawronski and Shawyer [11] proved that the distribution of the zeros of the Jacobi polynomials $P_{n}^{(a n+n, c n+d)}(x)$ follows an arcsine law. Gawronski and Shawyer pointed out that the latter result also follows from the earlier work of Mhaskar, Saff and Rahmaov.

In this work we did not attempt to apply Theorems 1 and 2 to the cases of the orthogonal polynomials of Wilson [19], Askey-Wilson [4], Racah [3], or Hahn [9].

\section{A DISCUSSION OF THEOREMS 2 AND 3}

We first indicate a proof of Theorem 3.

Proof of Theorem 3. If a satisfies (1.6) we define polynomials by (1.1) with $\beta_{n}=0, \alpha_{n}=\gamma_{n}=1 / 2$. In this case $p_{N+1}(x)=U_{N+1}(x)$, a Chebyshev polynomial of the second kind; that is, $U_{N+1}(x):=\sin [(N+2) \theta] / \sin \theta, x=$ $\cos \theta$. The extreme zeros of $p_{N+1}(x)$ are $\pm \cos [\pi /(N+2)]$. We now apply Theorem 1 with $-A=B=\cos [\pi /(N+2)]+\varepsilon, \varepsilon>0$, and conclude that $\left\{\left[4 \cos ^{2}(\pi /(N+2))+\varepsilon\right]^{-1}\right\}_{1}^{N}$ must be a chain sequence for every $\varepsilon>0$.

Conversely assume that $\{a\}_{1}^{N}$ is a finite chain sequence. Apply Theorem 1 with $\beta_{n}=0, \alpha_{n}=\gamma_{n}=\sqrt{a}, A=-1, B=1$. The zeros of $p_{N+1}(x)$ must lie in $(-1,1)$. In this case $p_{N+1}(x)$ is $U_{N+1}(x / 2 \sqrt{a})$, and its extreme zeros are $\pm 2 \sqrt{a} \cos [\pi /(N+2)]$. Demanding that the extreme zeros of $p_{N+1}(x)$ must belong to $(-1,1)$ establishes $(1.6)$, and the proof is complete.

It may be of interest to compare the bound $B$ of Theorem 2 with the bounds obtained by direct estimation of $\left\|A_{N}\right\|$. There is no loss of generality in assuming $\alpha_{n}=\gamma_{n+1}$. One can express $A_{N}$ as $\left(\beta_{n} \delta_{m, n}\right)+\left(\alpha_{m} \delta_{m, n-1}\right)+\left(\alpha_{n} \delta_{m, n+1}\right)$, $m, n=0,1, \ldots, N-1$. Thus

$$
\left\|A_{N}\right\| \leq C, \quad C:=\max \left\{\beta_{n}: 0 \leq n<N\right\}+2 \max \left\{\alpha_{n}: 0 \leq n<N-1\right\} .
$$

Since the largest zero of $P_{N}(x)$ is the largest eigenvalue of $A_{N}$, the constant $C$ on the right-hand side of (2.1) gives an upper bound of the largest zero of 
$P_{N}(x)$. Choose $a_{n}=1 / 4$ in Theorem 2 and observe that

$$
\left(C-\beta_{n}\right)\left(C-\beta_{n-1}\right) a_{n} \geq\left(\alpha_{n-1}\right)^{2}, \quad n=1, \ldots, N-1 .
$$

Thus $C \geq B$, and the bound $B$ of Theorem 2 is always sharper than the bound $C$ of (2.1).

Van Doorn [17] observed that Theorems 1 and 2 can be proved using the bounds given by Cassini ovals. Although he only stated his results for $N=\infty$, his proofs work for $N<\infty$ without change.

\section{LAgUeRre AND MeIXNeR POLYNOMIALS}

We first consider the (generalized) Laguerre polynomials. The coefficients in the three term recurrence relation in $(1.1)$ are $[8,16], \beta_{n}=2 n+\alpha+1$, $\alpha_{n}=n+1, \gamma_{n}=n+\alpha$. The zeros of Laguerre polynomials are positive since

$$
\frac{\alpha_{n-1} \gamma_{n}}{\beta_{n} \beta_{n-1}}=\frac{n(n+\alpha)}{(2 n+\alpha+1)(2 n+\alpha-1)}=\frac{n}{2 n+\alpha+1}\left(1-\frac{n-1}{2 n+\alpha-1}\right) \text {. }
$$

Choose $a_{n}=\mathrm{a}$ constant $=1 / a$,

$$
a:=4 \cos ^{2}(\pi /(N+1))+\varepsilon, \quad \text { for some } \varepsilon>0 .
$$

The quadratic equation $(1.5)$ is $x^{2}-2(2 n+\alpha) x+n(n+\alpha)(4-a)+\alpha^{2}-1=0$. Thus

$$
x_{n}, y_{n}=2 n+\alpha \pm \sqrt{1+a n(n+\alpha)} \text {. }
$$

It is clear that $x_{n}$ increases with $n$, hence

$$
B=2 N+\alpha-2+\sqrt{1+a(N-1)(N+\alpha-1)} .
$$

Thus we proved

Theorem 4. Let $L(N, \alpha)$ be the largest zero of a Laguerre polynomial $L_{N}^{\alpha}(x)$. Then

$$
L(N, \alpha)<2 N+\alpha-2+\sqrt{1+a(N-1)(N+\alpha-1)},
$$

where $a$ is as in (3.1). Furthermore the smallest zero $I(N, \alpha)$ of $L_{N}^{\alpha}(x)$ satisfies

$$
I(N, \alpha)>2 N+\alpha-2-\sqrt{1+a(N-1)(N+\alpha-1)} .
$$

Szegö [16, Theorem 6.31.2] gives the bound

$$
\eta<2 N+\alpha+1+\sqrt{(2 N+\alpha+1)^{2}+\frac{1}{4}-\alpha^{2}},
$$

which is weaker than (3.3) for $N \geq 1, \alpha>-1$ even if we take $a=4$. Szegö [16, Theorem 6.32] gives the sharper inequality

$$
\begin{aligned}
{[L(N, \alpha)]^{1 / 2}<(4 N+2 \alpha+2)^{1 / 2}-6^{-1 / 3}(4 N+2 \alpha+2)^{-1 / 6} i_{1}, } \\
\alpha>-1 \text { and }|\alpha| \geq 1 / 4,
\end{aligned}
$$

where $i_{1}$ is the smallest zero of the Airy function. Szegö also points out that the constant $6^{-1 / 3} i_{1}=1.85575 \ldots$ cannot be replaced by a smaller one.

The associated Laguerre polynomials of [5] (see also [13]) satisfy (1.1) with $\beta_{n}=2 n+2 c+\alpha+1, \alpha_{n}=n+c+1, \gamma_{n}=n+c+\alpha$. They do not satisfy a linear second order differential equation, but they satisfy a fourth order linear differential equation (not of Sturm-Liouville type), $[12,20]$. One can similarly prove the following theorem. 
Theorem 5. The largest zero $\eta$ and the smallest zero $\xi$ of an associated Laguerre polynomial of degree $N$ satisfy the inequalities

$$
\begin{aligned}
& \eta<2 N+2 c+\alpha-2+\sqrt{1+a(N+c-1)(N+c+\alpha-1)}, \\
& \xi>2 N+2 c+\alpha-2-\sqrt{1+a(N+c-1)(N+c+\alpha-1)} .
\end{aligned}
$$

The Meixner polynomials $\left\{m_{n}(x, \beta, c)\right\}$ are orthogonal with respect to a discrete measure with masses $j(x, \beta, c)$ located at the nonnegative integers,

$$
j(x, \beta, c)=c^{x}(\beta)_{x} / x !, \quad x=0,1, \ldots, 0<c<1,
$$

Chihara [7, p. 161]. In Chihara's normalization

$$
m_{n}(x, \beta, c)=(\beta)_{n 2} F_{1}\left(-n,-x ; \beta ; 1-c^{-1}\right) .
$$

One reason for using the normalization of [14], namely,

$$
M_{n}(x ; \beta, c):=\frac{(-1)^{n} c^{n / 2}}{\sqrt{n !(\beta)_{n}}} m_{n}\left(\frac{x \sqrt{c}}{1-c}\right),
$$

is that the $M_{n}$ 's are orthonormal since they satisfy (1.1) with coefficients given by

$$
\alpha_{n}=\gamma_{n+1}=\sqrt{(n+1)(n+\beta)}, \quad \beta_{n}=\sqrt{c} \beta+n(1+c) / \sqrt{c} .
$$

Another reason is that then $c \rightarrow 1^{-}$then $M_{n}(x, \beta+1, c)$ tends to orthonormal Laguerre polynomials with parameter $\beta$. Specifically

$$
m_{n}\left(\frac{x \sqrt{c}}{1-c} ; \beta, c\right) \rightarrow n ! L_{n}^{(\beta-1)}(x) \quad \text { as } c \rightarrow 1^{-} .
$$

Thus the zeros of $M_{n}(x ; \beta, c)$ converge to the corresponding zeros of $L_{n}^{(\beta-1)}(x)$. Ismail and Muldon [14] obtained the rate of this convergence as $c \rightarrow 1^{-}$. One would expect the zeros of $M_{n}$ to behave like the zeros of Laguerre polynomials of order $\beta-1$. Here we estimate the largest zero of $M_{N}(x ; \beta, c)$ for large $N$ using chain sequences. Let $a$ be as in (3.1). In the notation of Theorem 2 we have

$$
x_{n}, y_{n}:=\sqrt{c} \beta+\left(n-\frac{1}{2}\right) \frac{(1+c)}{\sqrt{c}} \pm \frac{1}{2 \sqrt{c}} \sqrt{(1+c)^{2}+4 a c n(n+\beta-1)} .
$$

By treating $n$ as a continuous variable in $[1, N-1]$ we see that

$$
\frac{d x_{n}}{d n}=\frac{1+c}{\sqrt{c}}+\frac{a(2 n+\beta-1) \sqrt{c}}{\sqrt{(1+c)^{2}+4 a c n(n+\beta-1)}} .
$$

Hence $B$ in (1.4) is $x_{N-1}$.

Theorem 6. Let $M(N, \beta)$ be the largest zero of $M_{N}(x ; \beta, c)$. Then we have (3.9)

$$
M(N+1, \beta)<\sqrt{c} \beta+\left(N-\frac{1}{2}\right) \frac{(1+c)}{\sqrt{c}} \pm \frac{1}{2 \sqrt{c}} \sqrt{(1+c)^{2}+4 a c N(N+\beta-1)},
$$

where $a$ is as in (3.1). Furthermore the bound in (3.9) is sharp in the sense that

$$
M(N, \beta) \approx \frac{(1+\sqrt{c})^{2}}{\sqrt{c}} N \quad \text { as } N \rightarrow \infty .
$$


Proof. The argument preceeding this theorem establishes (3.9), so we only need to prove (3.10). Consider a matrix $A_{N}$ of the form following (1.2) where the $\alpha$ 's, $\beta$ 's, and $\gamma$ 's are defined in (3.8). Let $\mathbf{e}_{1}, \mathbf{e}_{2}, \ldots, \mathbf{e}_{N}$ be the usual basis for $\mathscr{R}^{N}$. For an integer $k, k<N$, define a vector $\mathbf{X}=\left(\mathbf{e}_{N}+\mathbf{e}_{N-1}+\cdots+\right.$ $\left.\mathbf{e}_{N-k+1}\right) / \sqrt{k}$. Recall that the $\alpha_{n}$ 's, $\beta_{n}$ 's, and $\gamma_{n}$ 's are increasing functions of $n$. For fixed $k$ and sufficiently large $N$ it is easy to see that

$\left\|A_{N}\right\|^{2} \geq\left\|A_{n} \mathbf{X}\right\|^{2} \approx\left[(k-2)\left(2 \alpha_{N-3}+\beta_{N-2}\right)^{2}+2\left(\alpha_{N-2}+\beta_{N-1}\right)^{2}+\left(\alpha_{N-3}\right)^{2}\right] / k$.

Therefore

$$
\begin{aligned}
M(N, \beta) & =\left\|A_{N}\right\| \geq\left\|A_{n} \mathbf{X}\right\| \\
& \approx N \sqrt{\left(1-\frac{2}{k}\right)\left(2+\frac{1+c}{\sqrt{c}}\right)^{2}+\frac{2}{k}\left(1+\frac{1+c}{\sqrt{c}}\right)^{2}+\frac{1}{k}},
\end{aligned}
$$

which, by choosing $k$ large, proves that $\liminf M(N, \beta) / N \geq(1+\sqrt{c})^{2} / \sqrt{c}$. This and (3.9) prove (3.10).

\section{The Meixner-PollaczeK POlynomials}

The Meixner-Pollaczek polynomials are

$$
P_{n}^{\lambda}(x ; \phi):=e_{2}^{i n \phi} F_{1}\left(-n, \lambda+i x ; 2 \lambda ; 1-e^{-2 i \phi}\right), \quad \lambda>0, \pi>\phi>0 .
$$

The normalization we used in (4.1) is the Andrews-Askey normalization of [2]. Chihara [8] uses a different normalization and refers to these polynomials as the Meixner polynomials of the second kind [8, Chapter VI]. Szegö [16] calls them the Pollaczek polynomials (on an infinite interval). The Meixner-Pollaczek polynomials satisfy the three term recurrence relation

$2 x \sin \phi P_{n}^{\lambda}(x ; \phi)=(n+2 \lambda) P_{n+1}^{\lambda}(x ; \phi)-2(n+\lambda) \cos \phi P_{n}^{\lambda}(x ; \phi)+n P_{n-1}^{\lambda}(x ; \phi)$.

It is straightforward to establish the following theorem.

Theorem 7. The largest zero $\xi$ and the smallest zero $\eta$ of $P_{N}^{\lambda}(x ; \phi)$ are bounded as

$$
A<\xi<\eta<B
$$

where

$$
\begin{aligned}
& A=\left(N+\lambda-\frac{3}{2}\right) \cot \phi-\frac{1}{2} \sqrt{\cot ^{2} \phi+4(N-1)(N+2 \lambda-2) / \sin ^{2} \phi} \\
& B=\left(N+\lambda-\frac{3}{2}\right) \cot \phi+\frac{1}{2} \sqrt{\cot ^{2} \phi+4(N-1)(N+2 \lambda-2) / \sin ^{2} \phi}
\end{aligned}
$$

On the other hand one can choose

$$
a_{n}=n(n+\beta-1) /[(L(N, \beta-1)-2 n-\beta+\varepsilon)(L(N, \beta-1)-2 n-\beta-2+\varepsilon)]
$$

for $\varepsilon>0$ in (1.5a) and establish inequalities connecting the largest zero of a Meixner polynomial of order $\beta$, and the largest zero of a Laguerre polynomial of order $\beta-1$. Similarly the choice

$a_{n}=n(n+2 \lambda-1) /[(L(N, 2 \lambda-1)-2 n-2 \lambda+\varepsilon)(L(N, 2 \lambda-1)-2 n-2 \lambda-2+\varepsilon)]$ 
for $\varepsilon>0$ leads to an upper bound for the largest zeros of a Meixner-Pollaczek polynomial with parameters $\lambda$ and $\phi$. The results are

$$
\begin{aligned}
& M(N, \beta) \leq \sqrt{c} \beta+\left(N-\frac{3}{2}\right)(1+c) / \sqrt{c} \\
& +\frac{1}{2} \sqrt{(1+c)^{2} / c+4(L(N, \beta-1)-2 N-\beta-2)(L(N, \beta-1)-2 N-\beta-4)}
\end{aligned}
$$

and

$\eta \sin \phi \leq\left(N+\lambda-\frac{3}{2}\right) \cos \phi$

$$
+\frac{1}{2} \sqrt{\cos ^{2} \phi+4[(L(N, 2 \lambda-1)-2 N-2 \lambda-2)(L(N, 2 \lambda-1)-2 N-2 \lambda-4)]} .
$$

One can then substitute in (4.6) and (4.7) for $L(N, \beta-1)$ and $L(N, 2 \lambda-1)$ the left-hand side of the sharp bound (3.5) and obtain bounds for $M(N, \beta)$ and $\eta$.

\section{ZEROS OF JACOBI POLYNOMIALS $P_{n}^{\left(a_{n}, b_{n}\right)}(z)$}

Let $a_{n}>-1, b_{n}>-1$, and

$$
a_{n} /\left(a_{n}+b_{n}+2 n\right) \rightarrow a \quad \text { and } \quad b_{n} /\left(a_{n}+b_{n}+2 n\right) \rightarrow b \quad \text { as } n \rightarrow \infty \text {. }
$$

Using Sturmian argument, Moak, Saff, and Varga [15] proved the following theorem.

Theorem 8. Let $s_{n}$ and $r_{n}$, respectively, be the largest and smallest zeros of the Jacobi polynomials in the title of this section and assume that $a_{n}$ and $b_{n}$ satisfy (5.1). Set

$$
\begin{aligned}
& r_{a, b}:=b^{2}-a^{2}-\left[\left(a^{2}+b^{2}-1\right)^{2}-4 a^{2} b^{2}\right]^{1 / 2}, \\
& s_{a, b}:=b^{2}-a^{2}+\left[\left(a^{2}+b^{2}-1\right)^{2}-4 a^{2} b^{2}\right]^{1 / 2} .
\end{aligned}
$$

Then

$$
\lim _{n \rightarrow \infty} r_{n}=r_{a, b} \text { and } \lim _{n \rightarrow \infty} s_{n}=s_{a, b} .
$$

Furthermore, Moak, Saff, and Varga [15] proved that the zeros of the aforementioned Jacobi polynomials are dense in $\left[r_{a}, b, s_{a, b}\right]$.

Next we give a proof of (5.4). In the process of proving (5.4) we will obtain upper bounds (lower bounds) for the largest (smallest) zeros of the polynomials under consideration.

Let us first show that

$$
\limsup _{n \rightarrow \infty} s_{n} \leq s_{a, b}
$$

Proof of (5.5). The Jacobi polynomials $\left\{P_{n}^{\left(a_{N}, b_{N}\right)}(x)\right\}$ satisfy (1.1) with

$$
\begin{aligned}
\alpha_{n} & =\frac{2(n+1)\left(n+a_{N}+b_{N}+1\right)}{\left(2 n+a_{N}+b_{N}+1\right)\left(2 n+a_{N}+b_{N}+2\right)}, \\
\beta_{n} & =\frac{b_{N}^{2}-a_{N}^{2}}{\left(2 n+a_{N}+b_{N}\right)\left(2 n+a_{N}+b_{N}+2\right)},
\end{aligned}
$$


and

$$
\gamma_{n}=\frac{2\left(n+a_{N}\right)\left(n+b_{N}\right)}{\left(2 n+a_{N}+b_{N}\right)\left(2 n+a_{N}+b_{N}+1\right)} .
$$

Note that $\alpha_{n}, \beta_{n}$, and $\gamma_{n}$ depend on $N$. We choose the chain sequence in (1.5a) to be the constant sequence $\{1 / 4\}$, and we set

$$
\begin{aligned}
S_{N}(n):= & \frac{b_{N}^{2}-a_{N}^{2}}{\left(2 n+a_{N}+b_{N}-2\right)\left(2 n+a_{N}+b_{N}+2\right)}+\left(2 n+a_{N}+b_{N}\right)^{-1} \\
& \cdot\left[\left\{\frac{b_{N}^{2}-a_{N}^{2}}{\left(2 n+a_{N}+b_{N}-2\right)\left(2 n+a_{N}+b_{N}+2\right)}\right\}^{2}\right. \\
& \left.\quad+\frac{4 n\left(n+a_{N}\right)\left(n+b_{N}\right)\left(n+a_{N}+b_{N}\right)}{\left(2 n+a_{N}+b_{N}-1\right)\left(2 n+a_{N}+b_{N}+1\right)}\right]^{1 / 2} .
\end{aligned}
$$

From Theorem 2 we get

$$
s_{n} \leq \max \left\{S_{N}(n): 1 \leq n \leq N-1\right\} .
$$

Assume that the above maximum is attained at $n=n_{N}$, that is,

$$
S_{n}\left(n_{N}\right)=\max \left\{S_{N}(n): 1 \leq n \leq N-1\right\} .
$$

We claim that

$$
\liminf _{N \rightarrow \infty} n_{N} / N=1 \text {. }
$$

Let us assume that (5.6) is false. Then there exists $c, 0 \leq c<1$, and an infinite set $\Lambda$ of natural numbers such that $n_{N} / N \rightarrow c$ as $N \rightarrow \infty, N \in \Lambda$. In such case and in view of (5.1) one would easily see that

$$
\lim _{N \rightarrow \infty, N \in \Lambda} S_{N}\left(n_{N}\right)=\left(b^{2}-a^{2}\right) \delta^{2}+\left\{\left[\delta^{2}\left(a^{2}+b^{2}\right)-1\right]^{2}-4 \delta^{2} a^{2} b^{2}\right\}^{1 / 2},
$$

where

$$
\delta=1 /[c+(a+b)(1-c)] .
$$

For fixed $a$ and $b$ the function $s_{a x, b x}$ satisfies $s_{a, b}>s_{a x, b x}$ for $x>1$. Therefore

$$
\lim _{N \rightarrow \infty, N \in \Lambda} S_{N}\left(n_{N}\right)=s_{a \delta, b \delta}<s_{a, b}=\lim _{N \rightarrow \infty} S_{N}(N-1) .
$$

But from the definition of $n_{N}$ we find $S_{N}\left(n_{N}\right) \geq S_{N}(N-1)$, which contradicts (5.7). Thus (5.6) must be true and $S_{N}\left(n_{N}\right) \rightarrow s_{a, b}$ as $N \rightarrow \infty$, which implies (5.5).

Next we show

$$
\liminf _{n \rightarrow \infty} s_{n} \geq s_{a, b} .
$$

We use the idea in the proof of Theorem 6. Let $A_{N}$ be the $N \times N$ matrix whose $(j, k)$ entry $a_{j, k}$ is $\left(\alpha_{k} \gamma_{j}\right)^{1 / 2} \delta_{j, k+1}+\beta_{k} \delta_{j, k}+\left(\alpha_{j} \gamma_{k}\right)^{1 / 2} \delta_{k+1, j}, j, k=$ $0,1, \ldots, N-1$. Clearly if $n / N \rightarrow 1$ as $N \rightarrow \infty$ then

$$
\lim _{N \rightarrow \infty} a_{n, n+1}=\frac{1}{2}\left[\left(a^{2}+b^{2}-1\right)^{2}-4 a^{2} b^{2}\right]^{1 / 2} \text { and } \lim _{N \rightarrow \infty} a_{n, n}=b^{2}-a^{2} \text {. }
$$

On the other hand

$$
S_{N}=\left\|A_{N}\right\| \geq\left\|A_{N} \mathbf{X}\right\|
$$


for $\mathbf{X}$ on the unit sphere in $\mathscr{R}^{N}$. Now set

$$
M=\lfloor\sqrt{N}\rfloor,
$$

where $\lfloor a\rfloor$ is the integral part of $a$, and choose $\mathbf{X}=M^{-1 / 2} \mathbf{X}_{N}$, where $\mathbf{X}_{N}$ is a vector having ones in its last $M$ components and zeros in the remaining $N-M$ components. Therefore

$$
\begin{aligned}
\liminf _{N \rightarrow \infty} s_{N} & \geq \liminf _{N \rightarrow \infty}\left\|A_{N} \mathbf{X}_{N}\right\| \\
& =\liminf _{N \rightarrow \infty} M^{-1 / 2}\left\{-a_{N-M-2, N-M-1}+\sum_{n=N-M}^{N-1}\left(2 a_{n-1, n}+a_{n, n}\right)\right\} \\
& =b^{2}-a^{2}+\left[\left(a^{2}+b^{2}-1\right)^{2}-4 a^{2} b^{2}\right]^{1 / 2}=s_{a, b} .
\end{aligned}
$$

Hence (5.5) and (5.8) establish the second limit relation in (5.4), namely,

$$
\lim _{N \rightarrow \infty} s_{n}=s_{a, b} \text {. }
$$

Finally the first limit relation in (5.4) follows from (5.9), and

$$
P_{n}^{(\alpha, \beta)}(-x)=(-1)^{n} P_{n}^{(\beta, \alpha)}(x)
$$

Szegö [16, §4.1].

\section{REFERENCES}

1. W. Al-Salam, Characterization theorems for orthogonal polynomials, Orthogonal Polynomials, Theory and Practice (P. Nevai, ed.), NATO ASI series, C-294, Kluwer, Dordrecht, 1990, pp. 1-24.

2. G. Andrews and R. Askey, Classically orthogonal polynomials, in Polynômes Orthogonaux et Applications (C. Brezinski et al, eds.), Lecture Notes in Math., vol. 1171, Springer-Verlag, New York, 1985, pp. 36-62.

3. R. Askey and J. A. Wilson, A set of orthogonal polynomials that generalize the Racah coefficients or 6-j symbols, SIAM J. Math. Anal. 10 (1979), 1008-1016.

4. __ A set of orthogonal polynomials that generalize Jacobi polynomials, Mem. Amer. Math. Soc., no. 319, Amer. Math. Soc., Providence, RI, 1985.

5. R. Askey and J. Wimp, Associated Laguerre and Hermite polynomials, Proc. Roy. Soc. Edinburgh Sect. A 96 (1984), 15-37.

6. L.-C. Chen and M. E. H. Ismail, On asymptotics of Jacobi and Laguerre polynomials, SIAM J. Math. Anal. 22 (1991), 1442-1449.

7. T. S. Chihara, Chain sequences and orthogonal polynomials, Trans. Amer. Math. Soc. 104 (1962), 1-16.

8. _ An introduction to orthogonal polynomials, Gordon and Breach, New York, 1978.

9. A. Erdélyi, W. Magnus, F. Oberhettinger, and F. G. Tricomi, Higher transcendental functions, vol. 2, McGraw-Hill, New York, 1953.

10. W. Gawronski, Strong asymptotics and the asymptotic zero distributions of Laguerre polynomials $L_{n}^{(a n+\alpha)}$ and Hermite polynomials $H_{n}^{(a n+\alpha)}$, J. Approx. Theory (to appear).

11. W. W. Gawronski and B. Shawyer, Strong asymptotic and the limit distribution of the zeros of Jacobi polynomials $P_{n}^{(a n+\alpha, b n+\beta)}$, J. Approx. Theory (to appear).

12. W. Hahn, Über orthogonalpolynome mit drei parametern, Deutche. Math. 5 (1940-41), 273-278.

13. M. E. H. Ismail, J. Letessier, and G. Valent, Linear birth and death models and associated Laguerre polynomials, J. Approx. Theory 56 (1988), 337-348. 
14. M. E. H. Ismail and M. E. Muldoon, A discrete approach to monotinicty of zeros of orthogonal polynomials, Trans. Amer. Math. Soc. 323 (1991), 65-78.

15. D. Moak, E. Saff and R. Varga, On the zeros of Jacobi polynomials $P_{n}^{\left(\alpha_{n}, \beta_{n}\right)}$, Trans. Amer. Math. Soc. 249 (1979), 159-162.

16. G. Szegö, Orthogonal polynomials, Colloq. Publ., fourth ed., vol. 23, Amer. Math. Soc., Providence, R.I., 1975.

17. E. Van Doorn, Representation and bounds for zeros of orthogonal polynomials and eigenvalues of sign-symmetric tri-diagonal matrices, J. Approx. Theory 51, (1987), 254-266.

18. H. S. Wall and M. Wetzel, Quadratic forms and convergence regions for continued fractions, Duke Math. J. 11 (1944), 89-102.

19. J. Wilson, Some hypergeometric orthogonal polynomials, SIAM J. Math. Anal. 11 (1980), 690-701.

20. J. Wimp, Explicit formulas for the associated Jacobi polynomials and some applications, Canad. J. Math. 39 (1987), 983-1000.

Department of Mathematics, University of South Florida, Tampa, Florida 33620

Department of Mathematics, University of Central Florida, Orlando, Florida 32816 